In der Rubrik „Literatur kompakt" werden die wichtigsten Originalarbeiten aus der internationalen Fachliteratur referiert.

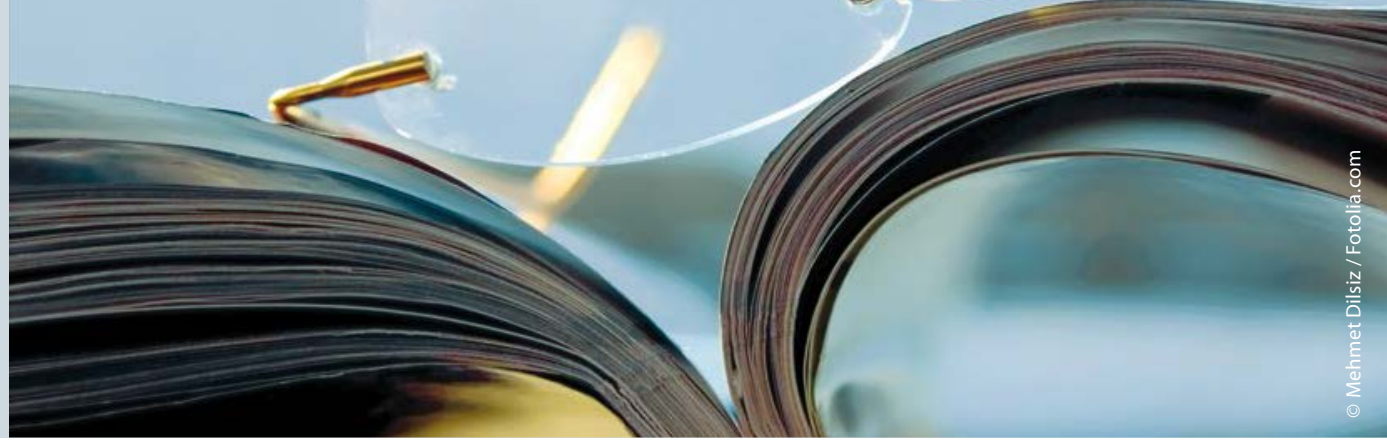

\section{Musik im OP mindert auch bei urologischen Eingriffen Angst und Schmerz}

Bei ambulanten urologischen Eingriffen lohnt es sich, angenehme Musik im Hintergrund zu spielen. Die Patienten sind dann weniger ängstlich und leider unter weniger Schmerzen. Darauf deutet eine Analyse von 16 kontrollierten Studien hin.

S elten freuen sich Patienten auf eine Prostatabiopsie oder eine Stoßwellenbehandlung, vielmehr fürchten sie sich vor den Schmerzen, möglichen Komplikationen und den Befunden. Musik im Hintergrund scheint da Wunder zu wirken. Die Patienten sind entspannter, haben deutlich weniger Angst und auch die Schmerzen sind geringer. Das geht jedenfalls aus einer systematischen Analyse von 16 Studien hervor.

Für ihre Analyse haben Forscher um Rena Kyriakides von der Uniklinik in Southampton, England, nur solche randomisiert-kontrollierten Untersuchungen berücksichtigt, die Gruppen mit und ohne Hintergrundmusik verglichen. Das Team um Kyriakides schaute dabei gezielt nach Studien, in denen primär die Auswirkungen auf Angst und Schmerzen geprüft wurden.

An den 16 geeigneten Studien hatten knapp 2.000 Patienten teilgenommen. Etwas mehr als zwei Drittel waren Männer. Bei der einen Hälfte der Teilnehmer wurde Hintergrundmusik gespielt, bei der anderen nicht. Alle Studien verwendeten eine visuelle Analogskala (VAS) zur Schmerzerfassung, Ängste wurden ebenfalls mit einer solchen Skala oder mit dem "State-Trait Anxiety Inventory (STAI)“ gemessen. $80 \%$ der Studien erschienen nach dem Jahr 2012.
In vier Studien mit knapp 290 Patienten spielte die Musik zu einer TRUS(transrektaler Ultraschall)-Biopsie, sechs Studien mit über 1.000 Patienten prüften Musik bei einer extrakorporalen Stoßwellenlithotripsie. Urodynamische Untersuchungen mit Hintergrundmusik wurden in zwei Studien mit 210 Patienten vorgenommen. Bei drei Studien wurde Musik während einer Zystoskopie (331 Patienten) und bei einer während der Verlegung eines Nierenkatheters abgespielt (100 Patienten).

Werden alle Studien zusammengefasst, so sind die Schmerz- und AngstScores bei ambulanten urologischen Eingriffen unter Musik signifikant geringer als ohne. In 14 Studien ergaben sich tendenziell oder signifikant geringere Schmerzen und Ängste, in acht Studien waren die Unterscheide für Schmerzen signifikant, in elf für Ängste. Bei neun Studien berichten die Forscher unter Musik eine höhere Zufriedenheit mit dem Eingriff, in sieben Studien waren die Patienten eher bereit, sich erneut einem solchen Eingriff $\mathrm{zu}$ unterziehen, wenn sie dabei Musik gehört hatten.

Fazit: Die Wissenschaftler um Kyriakides empfehlen die Studienergebnisse auch in Leitlinien stärker zu berücksichtigen. Dennoch seien auch potenzielle
Nebenwirkungen der Berieselung zu beachten. Nicht jedem Patienten gefällt die ausgewählte Musik, zudem kann es durch die Musik zu Kommunikationsproblemen zwischen Arzt und Patienten kommen - vor allem bei älteren Betroffenen mit Hörproblemen. Auch wenn Musik den meisten Patienten helfe, so müsse doch von Fall zu Fall entschieden werden, ob die Lautsprecher im OP anoder ausbleiben.

Thomas Müller

Kyriakides R, Jones P, GerAGHTY R, et al. Effect of music on outpatient urological procedures: A systematic review and metaanalysis from European Section of Uro-Technology (ESUT). J Urol 2017, https://doi.org/10.1016/j. juro.2017.11.117.

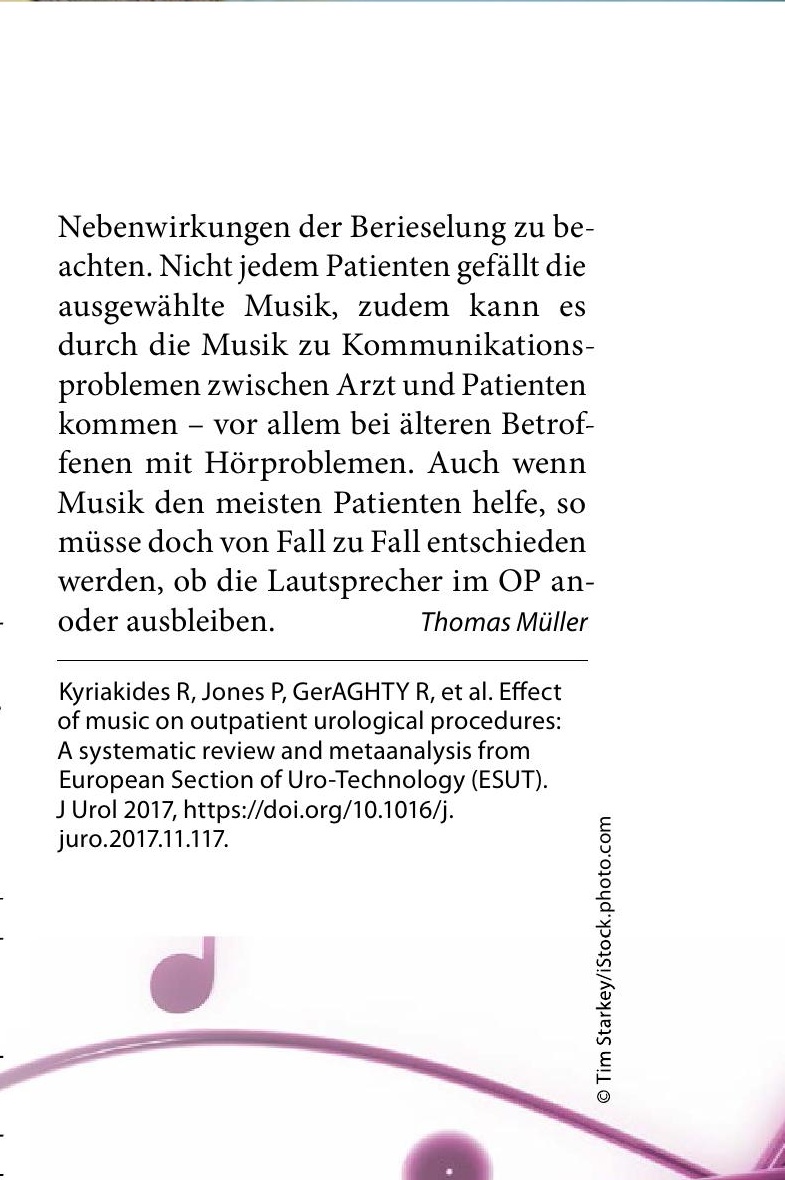

